\begin{abstract}
The release of chemical agents has the potential to cause loss of life and result in a major incident. Major incidents that result from the release of chemical agents will require a modified response by the emergency services due to the transmissibility, lethality, latency and persistence of chemical agents. In general, modifications to casualty flow, triage and treatment will have to be made in order to reduce the transmissibility and lethality of a chemical hazard. Within the context of an adapted response, emergency nurses must be familiar with the principles of care that relate to responding to a chemical incident that includes incident and casualty management as explained in this article.
\end{abstract}

\title{
Introduction
}

The first article of this two part series offered an overview of nerve agents and provided information relating to historical background, manufacture and the physiological effects of exposure. The focus of the current article builds on this foundation by considering the organophosphate biohazard and the management required following exposure. Pre-hospital care, triage and approaches to decontamination are discussed and subsequent in-hospital care is described. Important management issues including containment of contagious casualties and the critical need for effective psychological support are outlined.

\section{Organophosphate Hazards}

Organophosphate hazards may be encountered at home such as weed killer or in the livestock industry e.g. sheep-dip or found in nerve agents used by rogue governments and terrorist organizations (Beach 2010). The dangers they present can have varying magnitudes of effect, from fatalities to inconsequential symptoms (Calder \& Bland, 2018). The symptoms can be grouped into three categories: muscarinic effects, nicotinic effects, and central nervous system (CNS) effects. Two mnemonics to help remember the muscarinic effects are SLUDGE (salivation, lacrimation, urination, diarrhea, GI upset, and emesis) and DUMBELS (diaphoresis and diarrhea, urination, miosis, bradycardia, bronchospasm, bronchorrhoea, emesis, excess lacrimation, and salivation) (Katz, 2018). Nicotinic effects include muscle fasciculations, weakness, and diaphragmatic failure. The CNS effects range from anxiety to seizures and coma. The latter of which are commonly seen in children (Levy-Khademi, et. al., 2007) Given this wide 
range of effects, it is essential for clinicians to consider organophosphate exposure as a cause of symptoms. The toxicity, latency of onset of effects, and persistence of the effects are all functions of the chemical structure of the particular organophosphate. Those seen in accidental poisoning tend to have lower toxicity and do not persist for extended periods while those weaponized organophosphates have high toxicity and rapid onset of effects that persist for a longer period of time.

\section{Pre-hospital care}

The important first challenge in the management of exposure to terrorist incidents involving the use of nerve agents is the timely administration of antidotes. One of the effects of the nerve may be intense bronchoconstriction (this will depend on concentration level), thus the administration of atropine quickly, will reduce airway resistance and thus make airway management more straightforward (Sidell, 2003). Thus the ABCs should be adapted to AABCs with the first A covering antidote (Sidell, 1999). It is imperative that all nerve agent events must be contained and that effective decontamination conducted at the scene of the incident. Containment and decontamination at the scene will ameliorate the risks of spreading the toxin and contamination of local and regional treatment facilities. However, the pre-hospital care of those affected patients will place a tremendous strain on local emergency services such as police, fire and ambulance services (Kenar \& Karayilanoglu, 2004). In the United Kingdom, the medical response to a nerve agent attack is guided by the Major Incident Medical Management and Support (Hodgetts, 2003) protocol. This structured and standardized process gives the police primacy in conducting command, control and containment operations supported by specialist fire and ambulance teams that will conduct onsite decontamination. This multi-agency response requires significant investment in equipment and training that must be ongoing (Beach, 2010).

Both hospitals and smaller healthcare departments must be prepared to conduct containment and decontamination measures due to the possibility of patients bypassing the onsite decontamination facilities and travelling to hospital by ambulance or private car. Vehicles or treatment facilities that a victim may have visited prior to decontamination must be considered contaminated and unsuitable for use. In the mid-1990s, following the sarin release in the Tokyo subway, as many 
as $85 \%$ of patients bypassed Emergency Medical Services (EMS) and thus were not decontaminated (Veenema, 2007). Within this context, police have a vital part to play in controlling the flow of contaminated patients and isolating those people who are contaminated from unaffected individuals (Hodgetts, 2003).

\section{Triage}

Effective triage should be conducted as a priority action as the triage category is linked to prioritization for decontamination (Calder \& Bland, 2018). Ideally, triage can also be conducted along the casualty evacuation chain on arriving for decontamination. The role of decontamination is designed to limit the further absorption of chemical hazards such as nerve agents and permits healthcare professionals to deliver life-saving treatment (Beach, 2010). Effective decontamination also allows for the delivery of care in a clean and safe environment (Calder \& Bland, 2018). Currently, triage in the United Kingdom (UK) following release of a nerve agent follows the adapted chemical triage sieve categorization (NATO, 2018). This comprises four triage categories all preceded with the letter $\mathrm{T}$ to signify triage category listed below:

- T1 Immediate/Severe

- T2 Urgent/moderate

- $\quad$ T3 Delayed/minor

- T4 Expectant

The categories described allows for the standardization of triage criteria and are reproducible, swift and designed to take a systematic approach to assessing and delivering life-saving interventions that focus on airway, breathing and circulation (Calder \& Bland, 2018). The adapted chemical triage approach is diagrammatically summarized below.

Modified CBRN Triage Sieve 
Modified CBRN triage sieve

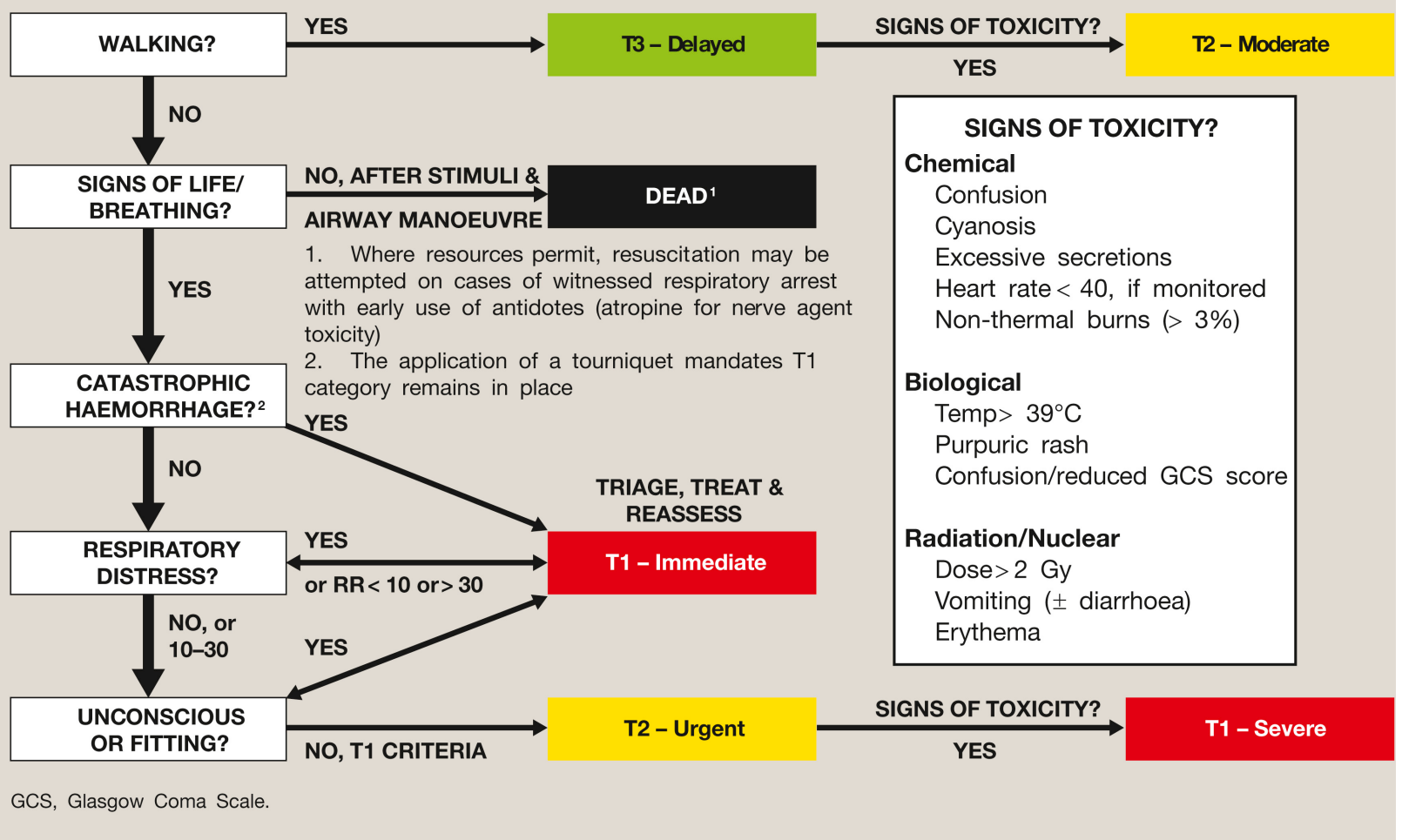

\section{Decontamination}

All staff who come into contact with patients of chemical agent contamination, equipment or vehicles must wear the most appropriate Personal Protective Equipment (PPE). This protocol will depend on the magnitude and type of attack (Beach, 2010). In the UK the preferred decontamination methods are:

- Disrobing

- Improvised dry decontamination

- Improvised wet decontamination

It should be noted that decontamination is not required if the chemical agent has been delivered as a gas due to the very quick half-life of a vaporized agent (Public Health England, 2018). 


\section{Dry Decontamination}

Dry decontamination must be conducted in the following way:

- Disrobing or undressing the victim is a highly effective way of reducing further exposure and ideally should occur within 15-20 minutes after exposure. Staff must consider the dignity and wellbeing of patients during this process, and every attempt should be made to provide shelter for individual patients.

- Use dry paper (e.g. blue roll, kitchen towel, toilet roll or paper tissues) to blot any exposed skin

- Use absorbent cloth material in order to avoid further body contamination avoiding vigorous blotting or friction

- All decontamination waste materials must be disposed of in the appropriate biohazard bag and stored in a well-ventilated space for disposal

(NHS Public Health England, 2018)

\section{Wet Decontamination}

Generally, wet decontamination should be conducted if the victim has signs and symptoms of contamination by a caustic substance (e.g. VX delivered as an oily liquid). Wet decontamination is the use of water from any available source such as taps, hose-reels, fixed hose reels and sprinkler systems (NHS Public Health England, 2018). The process for wet decontamination is detailed below:

- Optimal decontamination takes roughly 90 seconds when using water. Using a wash cloth with soap or detergent is also advised

- Use "RINSE-WIPE-RINSE" method of wet decontamination. Use with warm water and $5 \mathrm{mls}$ of detergent per liter of warm water

- Do not use bleach

- Self-decontamination by patients is the best approach, if possible. ED staff supervising

- WIPE affected areas of skin with absorbent material (cloth) carefully

- RINSE the decontaminated causality with clean warm water (no detergent) to remove the detergent and any residual chemicals and dry the skin with a clean towel 
- Hearing aids should be removed, but should not be immersed in water: either wipe thoroughly with saline-moistened gauze, place in clear plastic specimen bag and keep with victim. If the victim cannot hear without them, or place with other personal effects

- Eyes: if contact lenses present, remove if possible without harm; use topical anesthetic if needed; flush eyes copiously with $0.9 \%$ saline

- Contain waste water where possible: if not possible do not delay or defer decontamination, seek advice, and inform Environmental Protection Authorities and local utility companies.

(NHS Public Health England, 2018)

\section{In-hospital care}

Generally and optimally, casualties should be fully decontaminated in the pre-hospital phase (Calder \& Bland, 2018). However, there are occasions when more serious casualties are extracted from the scene before decontamination has occurred due to the severity of their injuries or possibly due to a delay in recognizing the causative agent. The latter point underscores the potential risk of non-decontaminated casualties walking into or self- referring to the Emergency Department (ED). In order to respond effectively EDs must have enough appropriately qualified staff on duty to recognize, assess, and treat casualties following release of a nerve agent.

Signs such as pinpoint pupils, muscle twitching, confusion, chest tightness, sweating, rhinorrhoea and bronchorrhoea are clinically important. Advice and assistance should be sought at an early stage from the most locally appropriate Poisons/Hazardous materials information service. Multiple sites for Intravenous access will be required as a priority. This is preferable in order to avoid incompatibilities that may arise if multiple drugs are administered through the same cannula (Vale et al, 2018). The primary drug treatment is atropine, and patients should receive $2 \mathrm{mg}$ IM or IV and repeated every 5 minutes as needed until bradycardia, bronchorrhoea, and bronchospasm resolve. If there is a partial response to $2 \mathrm{mg}$, the dose can be increased to 4 $\mathrm{mg}$ (Vale et al, 2018). The atropine will block the effect of acetylcholine. The heart rate, blood pressure, pupil size as well as lung sounds should be recorded before and with each dose. If available within minutes to a few hours after exposure, an "oxime" such as pralidoxime (2PAM), obidoxime or HI-6 should be administered after giving atropine. 1-2 grams of pralidoxime IV (250/5 mins) until symptoms improve. Alternatively, three doses of $600 \mathrm{mg}$ may be given in rapid succession. The 3-dose regiment may be repeated in 1 hour. Supportive care 
includes administration of $100 \%$ oxygen via a non-rebreathing mask at 15 liters, if able to maintain airway, the use of diazepam to prevent or manage seizures (this is also is useful for its anxiolytic properties), and albuterol for bronchospasm. If there is severe bronchorrhea, endotracheal intubation and positive pressure ventilation may be necessary. Fluid and electrolytes should be replaced as appropriate.

\section{Containment of Contagious Casualties}

There may be situations following decontamination, where casualties still present a hazard. If casualties have become contaminated whilst in hospital or are still contagious (off gassing) they will require to be isolated (Calder \& Bland, 2018). All in hospital patient movements should be kept to a minimum with respiratory protection provided to patients to ameliorate aerosol contamination (Beach, 2010).

\section{Psychological Support}

ED nurses must be prepared for potentially highly anxious and emotional patients who have or think they have been patients of nerve agent poisoning. Pre-incident training consisting of vignettes, exercises and drills can help prepare the team. When reports of a local nerve agent incident emerge, an initial step is to conduct (or get a member of the team to conduct) a quick review of the media coverage (including social media). This will help ascertain an initial insight into the community perceptions of the event and illustrate the local commentary regarding risk and potential public anger. It is often the media coverage of painful death that lead to anxiety, panic, fear and the associated symptomology.

ED attendees may be crying or screaming, with some believing they may die. There may be irrational fears such as a concern that they might infect a partner or family. The result will be signs of raised autonomic arousal, such as breathing difficulties, perspiration, light-headedness, nausea or shaking, or reporting cardiac palpitations (NHS Choices, 2018). Certain symptoms such as pinpoint pupils, sweating and mild to moderate difficulty breathing may also be due to 
panic rather than being attributable to the effects of a nerve agent. ED nurses should attempt to quickly determine which symptoms are related to fear and which potentially to nerve agent poisoning. The ED nurses must to be cognisant of potential additional stressors if friends or family were in the vicinity of the event. In addition, ED nurses are also likely to be faced with equally anxious family members.

From a MH perspective, when dealing with a patient who has been / may have been exposed to a nerve agent, then good communication (both verbal and nonverbal) is imperative. Patients who are fully conscious and have full muscular control will require minimal nursing care but their fear will be real (HHS, 2018a). Therefore, all staff need to be aware of the MH requirement and certain aspects of good practice to help the patient and maintain relative calm McGhee et al, 2015) The following initial management is recommended: In the ED nurse dealing with individuals who may have been exposed to or affected by nerve agent poising should consider the following initial psychological support

- $\quad$ Place the patient in a comfortable sitting position.

- Keep calm, and give a clear and consistent message.

- Speak clearly, avoid jargon, sound confident, and maintain good eye contact.

- If the patient is panicking, or hyperventilating, encourage him or her to take deep and slow breaths, and praise their efforts.

- If the patient has a dry mouth, give him or her water.

- Explain what the assessment or treatment involves but do not overwhelm the patient with information. If appropriate, tell the patient that there is period of observation and what this entails.

- Be positive about outcomes but keep to the facts. Avoid negative messages and do not speculate.

- Provide plenty of opportunities for patients to ask questions and acknowledge his or her concerns.

- After you have explained the treatment, ask the patient to confirm that he or she understands what has been said.

- If prepared, provide a patient information leaflet which ED attendees can take home. Example. (HHS, 2018b) 
- If the patient is to be discharged, ensure he or she is calm and relaxed if driving home. With his or her permission, contact a friend or relative if appropriate.

\section{Conclusion}

Managing patients who have been exposed to organophosphates requires a high index of suspicion by health care providers to take necessary precautions to prevent exposure to themselves and others while performing triage and treatment. Decontamination is important to decrease the persistence of the effects and minimize the risk of exposing others. While administering medications to mitigate the effects of organophosphates is essential, attending to the psychological needs of patients is equally important. 


\section{References}

Beach, M. (2010). Disaster Preparedness and Management. Philadelphia, PA: F.A. Davis.

Calder, A, \& Bland, S. (2018) CBRN considerations in a major incident, Surgery (36), 8, 417423.

Hodgetts, T. J. (2003). "Major Incident Medical Training: A Systematic International Approach." International Journal of Disaster Medicine 1(1): 13-20.

Katz KD. (2018). Organophosphate toxicity. http://emedicine.medscape.com/article/167726$\underline{\text { clinical }}$

Kenar L, Karayilanoglu T. (2004) Pre- hospital management and medical intervention after a chemical attack. Emergency Medicine Journal;21(1):84-8

Levy-Khademi F, Tenenbaum AN, Wexler ID \& Amaitai Y. (2007). Unintentional organophosphate intoxication in children. Pediatric Emergency Care, 23, 716-718.

McGhee, S., Finnegan, AP., Clochesy, J., \& Visovsky, C. (2015). Snakebite: Not Just a Tropical Problem. A Guide for Emergency Nurses. Emergency Nurse, 22(9) pp 24 - 29

North Atlantic Treaty Organization (2018) Allied Medical Publication (AMedP) 7.1 (EditionA): The Medical Management of CBRN Casualties. NATO, Brussels

NHS Choices (2018). Panic Disorders: Symptoms. At https://www.nhs.uk/conditions/panicdisorder/\#symptoms Accessed 2 August 2018

NHS Public Health England (2018). Chemical, biological, radiological and nuclear incidents: clinical management and health protection.

https://assets.publishing.service.gov.uk/government/uploads/system/uploads/attachment_data/file 1712888/Chemical_biological_radiological_and_nuclear_incidents_clinical_management and $\mathrm{h}$ ealth protection.pdf Accessed 4 September 2018

Sidell, F. (1999) Features. Agents of the Month: Nerve Agents (http://chembio.janes.com)

Sidell, F. (2003) Nerve agents. In: Biggs, S.M. (E.d), Advanced Disaster Medical Response Manual for Providers. Harvard Medical International, Boston, USA. Pp.62-63.

US Department of Health and Human Services (2018a) Chemical Hazards Emergency Medical Management. Nerve Agents - Emergency Department/Hospital Management. At: https://chemm.nlm.nih.gov/na hospital_mmg.htm Accessed 30 July 2018 
US Department of Health and Human Services (2018b) Agency for Toxic Substances and Disease Registry (ATSDR). https://www.atsdr.cdc.gov/MHMI/mmg166-handout.pdf\#page=1 Accessed 30 July 2018

Vale, A Mars T.C. Maynard, R. (2018): Novichok: a murderous nerve agent attack in the UK, Clinical Toxicology, DOI: 10.1080/15563650.2018.1469759

Veenema TG, ed. (2007) Disaster Nursing and Emergency Preparednessfor Chemical, Biological and Radiological Terrorism and Other Hazards.2nd ed. New York, NY: Springer. 